\title{
Evaluation of Distraction Effect of Music Stimuli during Cycling Exercise with Low intensity in terms of Multiple Time Scale
}

\author{
Caijilahu Bao, Zhiqiang Ma, Member, IEEE, and Tohru Kiryu, Member, IEEE
}

\begin{abstract}
There is a lack of research on the distraction effects of music stimuli with the multiple time scales of biological functions. It should be preferable to propose an effective strategy for evaluating the time varying behavior of psychological responses to music during exercise on autonomic nervous activity . From the RR interval time series of electrocardiograms recorded during cycling with low intensity, we estimated autonomic nervous activity related indices: time and frequency domain indices estimated from the fluctuations of the RR interval. Then we searched the temporal distribution of the stimulus response module appeared in the time varying behavior. The inspection of distraction effects was done for properly selected autonomic nervous activity related indices and the ratings of perceived exertion for evaluating appropriate participants based on both the impression of favorability to music and the stimulus response module occurrences. The results inferred that the multiple time scales strategy could be of help to assess the suitable approach to identify the distraction effects of music stimuli.
\end{abstract}

Index Terms-distraction effect, music stimuli, multiple time scale, perceived exertion.

\section{INTRODUCTION}

A $\mathrm{N}$ effect of listening music on different psychological functions has been discussed in terms of relaxation and refreshment in stressful situations [1], [2], [3], [4], [5]. These findings support that listening to music could change the focus of attention but could not change the physiological fatigue [6].

Regarding the relationship between cycling exercise and simultaneous fatigue responses, Hayano et al. reported that autonomic nervous activity (ANA) regulates physical activity levels based on cardiorespiratory interaction during cycling [7]. Kiryu et al. studied the overall behavior of the heart rate (HR) during cycling and rest periods in relation to the cardiorespiratory cycle, the muscle contractions, and the ratings of perceived exertion (RPE) under different workload intensities [8]. On the basis of the current interpretation of the modulating effects, music is perceived as a diversion from unpleasant proprioceptive sensations that arise from exhaustion. However, the different distractive effects reported in the above studies have made their interpretation uncertain. The disparities between these studies could be due to many factors,

This work was partially supported by National Natural Science Foundation of China $(61762070,61862048)$ for exploratory research.

C. Bao and Z. Ma are with the Department of College of Data Science and Application, Inner Mongolia University of Technology, Hohhot, China, 010080 China e-mail: (bao-caijilahu@ 163.com)

T. Kiryu, was with the Department of Graduate School of Science and Technology, Niigata University, Niigata, Japan, 950-2181 Japan e-mail: (kiryu@eng.niigata-u.ac.jp). among which are the heterogeneity of study populations, interventions, and outcome measures etc. Moreover, there is a lack of research on the distractive effects of music with multiple time scales that should be applied to identify the most effective strategy for assessing them mainly via ANA.

Accordingly, we will adopt time varying behavior (TVB) of ANA-related indices and multivariate analysis investigating the physiological and psychological responses to music stimuli during cycling. Our purpose is to conduct a feasibility study on a multiple time scales strategy that could clarify the distractive effects by determining suitable relationships among participants and physiological and psychological assessments.

\section{Methodes}

\section{A. Participants}

Fifteen healthy males $(23.1 \pm 2.6$ years old $)$ provided written informed consent to their participation in all procedures associated with the study. They reported that they had not received formal musical education and not played any musical instrument within the last five years. They indicated to what extent they usually listen to music during walking or running. The experimental procedure and tasks were explained; details regarding with the aims of the study or the variables of interest were omitted. Prior to the experiments, the ethical approval was granted by the ethics committee at Niigata University.

\section{B. Experimental procedure}

By placing the surface electrode (Bs-150, Nihon Kohden Inc.) on the chest (V6), we recorded electrocardiogram (ECG) signals at a sampling frequency of $1000 \mathrm{~Hz}$ (Myomonitor IV, Delsys Inc.) for a cycling (60 rpm) trial with a bicycle ergometer (STB-1400, Combi Inc.). The workload was constant low intensity cycling exercise (50 watts) [9]. The music stimulus was jazz with a 135-bpm tempo, played through earphones and adjusted to a comfortable level $(70-85 \mathrm{~dB})$.

The sequence (Fig. 1) took a total of 22 minutes per trial: a participant performed a 5-min warm up of cycling without music stimuli and then took a 1-min rest to regulate HR; five consecutive 3-min cycling tasks (T\# ) were then carried out while listening to music (T1 to T5; total 15 minutes); The participants were given a questionnaire during each minute of the task, in which they were asked to rate their RPE. The last, to evaluate the music impression, participants completed a 1-min simple questionnaire after the cycling exercise. We combined the five tasks into three overlapping phases (Fig. 


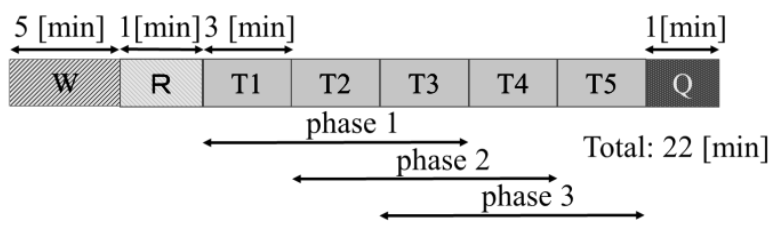

W: warm up, R: rest, T \#: task \#, Q: questionnaire

Fig. 1. Experiment sequence.

1): phase 1 ( $\mathrm{T} 1, \mathrm{~T} 2$, and $\mathrm{T} 3$ ), phase $2(\mathrm{~T} 2, \mathrm{~T} 3$, and $\mathrm{T} 4)$, and phase 3 (T3, T4, and T5), shifting phases every $3 \mathrm{~min}$.

\section{Biosignal processing}

In the psychological questionnaire, the participants selected the appropriate RPE displayed in the panel on the handle every minute: Borg's RPE ranging from 6 to 20 [10]. The music impression was defined on a scale ranging from 0 to 5 for 26 categories [11]; the total score ranged between 0 and 130 .

For biosignal processing in the TVB of the psychological responses, we used a resampling $(4 \mathrm{~Hz})$ algorithm with cubic spline interpolation to obtain uniformly sampled R-R interval (RRI) time series. For the fluctuation of RRI, i.e., heart rate variability (HRV), as the time domain index, we estimated the total RRI, the mean RRI, the standard deviation of the RRI (SDNN), the root mean square of successive RRI differences (RMSSD), and the SDNN/RMSSD ratio that would play a relative indicator of the sympathetic nervous activity[12], [13]. Estimating the frequency domain indices, we used a continuous wavelet transform with a Gabor function controlling the time and frequency resolution. They were the low-frequency (LF) $(0.04-0.15 \mathrm{~Hz})$ and high-frequency (HF) $(0.15-0.45 \mathrm{~Hz})$ components as well as the LF/HF ratio, as the conventional indicators of ANA [14]. The ANA-related indices were then evaluated in the time and frequency domains every $10 \mathrm{~s}$ : the total number of samples was 90 for the five consecutive tasks. We introduced non-uniformly sampled TVB of ANArelated indices for a stimulus response module (SRM) (Fig. 2) that could be composed of stimuli as a trigger point and the response as an accumulation section (AS) [15]. In the expected physiological meaning, the duration is short for stimuli, and longer for response. When stimuli occur frequently with in the duration of response, the responses superimposed in time. This could be defined as an accumulation of physiological responses. Therefore, the occurrence of SRM indicates would be higher in the participants with favorability for stimulating music. The expected AS was surveyed based on the following SRM-related conditions: normalized LF component greater than $120 \%$ (LF120) and normalized HF component less than 80\% (HF80) [15], [16]. The onset for AS (over a 2-s epoch) was estimated by tracing the TVB of the normalized LF component backwards in time for locating the local minimum, as the time of the trigger point. Note that the indices were normalized by each mean for $3 \mathrm{~min}$ in the middle of the warm up. The measured data was analyzed using Matlab R2014a (MathWorks Inc.) to estimate the physiological indices.

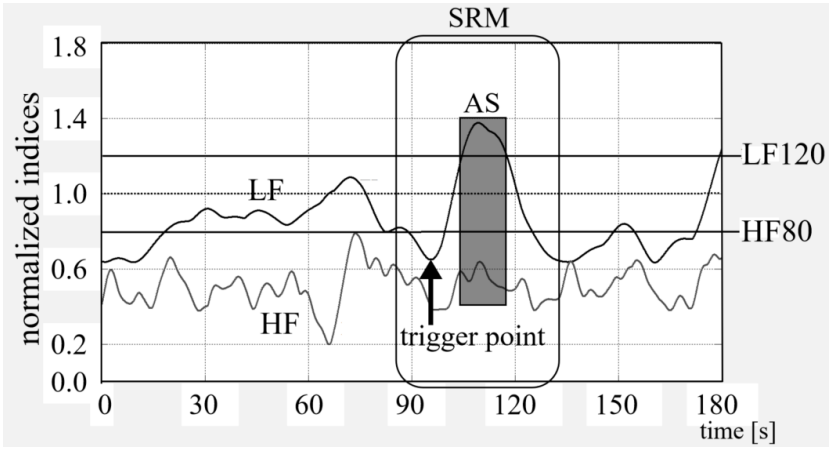

Fig. 2. Definition of a SRM in time varying behavior of LF and HF components.

\section{Statistical analysis}

The participants were divided into two groups based on the median of the total score ("favorite:" score $\geq$ median; "nonfavorite:" score < median). Then, the differences between two groups were evaluated by multiple regression analysis and factor analysis to evaluate the distraction effect of the stimulating music during exercise. The statistical analyses were performed using JMP 11 (SAS Institute Inc.).

In the multiple regression analysis, we used the ANArelated indices as explanatory variables to estimate the psychological index, RPE. Furthermore, to evaluate the distraction effect we performed a correlation analysis to confirm the strong correlation between HR and RPE ( $\gamma$ HR-RPE) [10]. In the factor analysis, the number of common factors on the physiological indices was determined as follows: factors whose eigenvalue was greater than 1.0 [17] were only retained for the variable loadings along with over $70 \%$ cumulative contribution ratios. To bivariate normal distribution was performed for each pair of factor score samples, and then a nonparametric density profile was observing for overlapping three phases in the two groups.

\section{RESUlts}

Based on the music impression score, the participants (15) were divided into favorite (8) and non-favorite (7) groups by the median of music impression score, 82 (Fig. 3).

Furthermore, by observing the TVB of ANA-related indices, there were temporal distributions of 19 SRMs: 14 in favorites and 5 in non-favorites. As a result, the number of occurrences in the favorite group was more than that in the non-favorite one and the occurrences were more concentrated in phase 3 than in phases 1 or 2 , that is, later in a trial (Fig. 4).

Figure 5 depicts the TVBs of the measured RPE (mRPE) and the estimated RPE (eRPE) with the ANA-related indices employed in multiple regression equation. The mRPE showed it linearly increases until experimental segment $\mathrm{T} 4$ and tends to saturate at T5 in each group, and this trend was more explicit in eRPE. Moreover, the measured RPEs in the non-favorite group from T2 to T5 significantly (* p $<0.05$, ** $\mathrm{p}<0.01$ ) increased more than those in the favorite group (Fig. 6).

The correlation coefficient decreased from $\mathrm{T} 1$ to $\mathrm{T} 3$ and then increased from $\mathrm{T} 3$ to $\mathrm{T} 4$, after which the correlation coefficient becomes small or no correlation or inverse correlation 


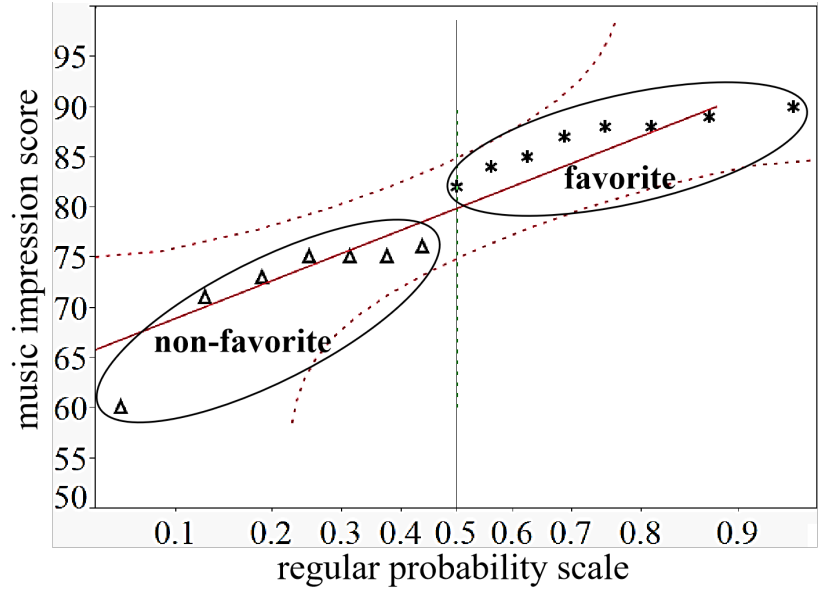

Fig. 3. The participants were divided into favorite and non-favorite groups by the median of music impression score.

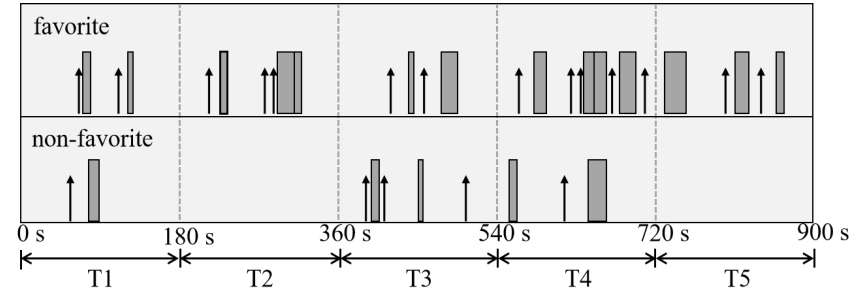

Fig. 4. Time distribution of SRM in favorite and non-favorite groups.

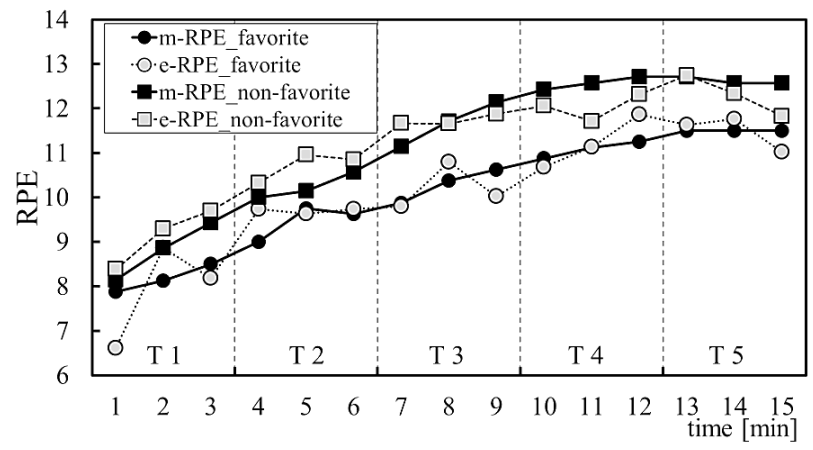

Fig. 5. TVB of measured and estimated RPE in each group during $15 \mathrm{~min}$ cycling.

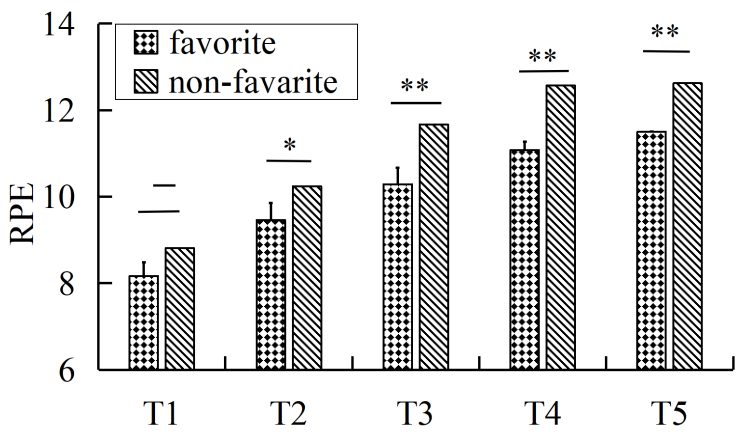

Fig. 6. Comparison of RPE between favorite and non-favorite group during consecutive each tasks (- not significantly).
TABLE I

CORRELATION COEFFICIENT OF HR AND RPE (MEASURED AND ESTIMATED) AT EACH TASK ON EACH GROUP.

\begin{tabular}{c|cccccc}
\hline$\gamma$ HR-RPE & group & T1 & T2 & T3 & T4 & T5 \\
\hline$\gamma$ HR-mRPE & favorite & 0.94 & 0.79 & 0.29 & 0.99 & - \\
& non-favorite & 0.99 & 0.88 & -0.1 & 0.52 & 0.43 \\
\hline$\gamma$ HR-eRPE & favorite & 0.83 & -0.02 & -0.76 & 0.95 & -0.66 \\
& non-favorite & 1.00 & 0.89 & 0.51 & 0.99 & -0.14 \\
\hline
\end{tabular}

TABLE II

FACTOR STRUCTURE FOR ANA-RELATED INDICES AT THREE PHASES.

\begin{tabular}{c|cccc}
\hline group & phase \# & factor \# & eigenvalue & $\begin{array}{c}\text { cumulative } \\
\text { contribution ratio[\%] }\end{array}$ \\
\hline \multirow{4}{*}{ favorite } & phase 1 & factor 1 & 5.23 & 0.65 \\
& & factor 2 & 1.23 & 0.81 \\
& phase 2 & factor 1 & 3.47 & 0.43 \\
& & factor 2 & 2.36 & 0.73 \\
& phase 3 & factor 1 & 3.04 & 0.38 \\
& & factor 2 & 2.52 & 0.70 \\
\hline \multirow{4}{*}{ non-favorite } & phase 1 & factor 1 & 5.38 & 0.67 \\
& & factor 2 & 1.27 & 0.83 \\
& \multirow{2}{*}{ phase 2 } & factor 1 & 4.30 & 0.54 \\
& & factor 2 & 2.01 & 0.79 \\
& \multirow{2}{*}{ phase 3 } & factor 1 & 4.30 & 0.54 \\
& & factor 2 & 2.12 & 0.80 \\
\hline
\end{tabular}

at T5 (Table 1). Moreover, except for the $\gamma \mathrm{HR}-\mathrm{mRPE}$ on T3 and $\mathrm{T} 4$, the correlation coefficient of the favorite group was smaller than that in the non-favorite group on each task, and this phenomenon was more obvious on the $\gamma$ HR- eRPE.

In factor analysis, the two common factors, factor 1 and 2, were determined on the basis of the factor structure (Table 2) and factor loading (Table 3) of the ANA-related indices in the three phases.

In the favorite group, the time domain indices, RRI and $\mathrm{HR}$, were active on factor 1 , but not active on factor 2 during the three phases; SDNN and SDNN/RMSSD were active on factor 2 in phase 2, but not active during any of the other phases. The frequency domain indices, LF and LF/HF, were active on factor 2 during phases 1 and 3; HF was active on factor 2 in phase 3 . In the non-favorite group, the time domain indices, RRI and HR, were active on factor 1 in phases 1 and 3. SDNN and SDNN/RMSSD were active on factor 1 in phase 2, while RMSSD was active on factor 2 in phase 1 . The frequency domain index, LF, was active on factor 2 in phase 3, while HF was active on factor 2 in phases 1 and 3. Figure 7 shows the bivariate normal ellipse distribution of score samples for each pair of factors. In the favorite group, the scores are rather concentrated on the first factor in phase 3 and less on the second factor than that for other phases. In the non-favorite group, from the nonparametric density, the scores were probably normally distributed on the first and second factors for the three phases.

\section{DISCUSSION}

\section{A. Evaluation of distraction effects with SRM in TVB of ANA- related indices}

Time domain measures were adopted to study sympathovagal balance during low intensity cycling exercise [13]. They were particularly important in the context of HRV monitoring 
TABLE III

FACTOR LOADINGS FOR ANA-RELATED INDICES.

\begin{tabular}{|c|c|c|c|}
\hline \multirow[t]{2}{*}{ group } & phase \# & phase 1 & \\
\hline & indices & factor 1 & factor 2 \\
\hline \multirow{8}{*}{ favorite } & RRI & 0.93 & 0.28 \\
\hline & HR & -0.94 & -0.24 \\
\hline & LF & 0.50 & 0.78 \\
\hline & $\mathrm{HF}$ & 0.50 & 0.60 \\
\hline & $\mathrm{LF} / \mathrm{HF}$ & 0.11 & 0.72 \\
\hline & SDNN & 0.58 & 0.31 \\
\hline & RMSSD & 0.69 & 0.29 \\
\hline & SDNN/RMSSD & 0.01 & 0.10 \\
\hline \multirow{8}{*}{$\begin{array}{l}\text { non- } \\
\text { favorite }\end{array}$} & RRI & 0.88 & 0.43 \\
\hline & HR & -0.88 & -0.43 \\
\hline & LF & 0.58 & 0.62 \\
\hline & $\mathrm{HF}$ & 0.44 & 0.74 \\
\hline & $\mathrm{LF} / \mathrm{HF}$ & 0.11 & 0.15 \\
\hline & SDNN & 0.39 & 0.62 \\
\hline & RMSSD & 0.52 & 0.84 \\
\hline & SDNN/RMSSD & 0.06 & 0.00 \\
\hline \multirow{2}{*}{ group } & phase \# & phase 2 & \\
\hline & indices & factor 1 & factor 2 \\
\hline \multirow{8}{*}{ favorite } & RRI & 0.95 & -0.02 \\
\hline & HR & -0.96 & 0.02 \\
\hline & LF & 0.04 & 0.17 \\
\hline & $\mathrm{HF}$ & 0.7 & 0.22 \\
\hline & $\mathrm{LF} / \mathrm{HF}$ & -0.36 & 0.18 \\
\hline & SDNN & 0.20 & 0.80 \\
\hline & RMSSD & 0.40 & -0.10 \\
\hline & SDNN/RMSSD & -0.19 & 0.90 \\
\hline \multirow{8}{*}{$\begin{array}{l}\text { non- } \\
\text { favorite }\end{array}$} & RRI & 0.08 & -0.88 \\
\hline & HR & -0.07 & 0.89 \\
\hline & LF & 0.38 & -0.12 \\
\hline & $\mathrm{HF}$ & 0.29 & -0.25 \\
\hline & LF/HF & 0.18 & 0.40 \\
\hline & SDNN & 0.90 & -0.22 \\
\hline & RMSSD & -0.05 & -0.35 \\
\hline & SDNN/RMSSD & 1.00 & 0.02 \\
\hline \multirow[t]{2}{*}{ group } & phase \# & phase 3 & \\
\hline & indices & factor 1 & factor 2 \\
\hline \multirow{8}{*}{ favorite } & RRI & 0.97 & -0.03 \\
\hline & HR & -0.98 & 0.03 \\
\hline & LF & -0.02 & 0.85 \\
\hline & $\mathrm{HF}$ & 0.18 & 0.85 \\
\hline & $\mathrm{LF} / \mathrm{HF}$ & -0.29 & 0.75 \\
\hline & SDNN & 0.22 & 0.20 \\
\hline & RMSSD & 0.41 & 0.21 \\
\hline & SDNN/RMSSD & -0.15 & 0.09 \\
\hline \multirow{8}{*}{$\begin{array}{l}\text { non- } \\
\text { favorite }\end{array}$} & RRI & 0.98 & -0.10 \\
\hline & HR & -0.97 & 0.11 \\
\hline & LF & -0.12 & 0.83 \\
\hline & $\mathrm{HF}$ & -0.16 & 0.84 \\
\hline & $\mathrm{LF} / \mathrm{HF}$ & -0.01 & 0.34 \\
\hline & SDNN & -0.10 & 0.51 \\
\hline & RMSSD & 0.21 & 0.35 \\
\hline & SDNN/RMSSD & -0.22 & 0.29 \\
\hline
\end{tabular}

in real time or ambulatory settings. Moreover, to characterize the autonomic response to musical stimuli, biosignal processing in time and frequency domain has been simultaneously provided [18].

Regarding the autonomic responses, spectral analysis appeared to demonstrate more sensitive and enlightening HRV indices compared with time domain analysis [19]. Thus, we surveyed the occurrences of SRM in the TVB of the frequency domain indices to verify the time distribution of physical fatigue related autonomic regulation (Fig. 4). Since the LF and HF components stem from Mayer waves in blood pressure and respiratory sinus arrhythmia in respiration [7], they are
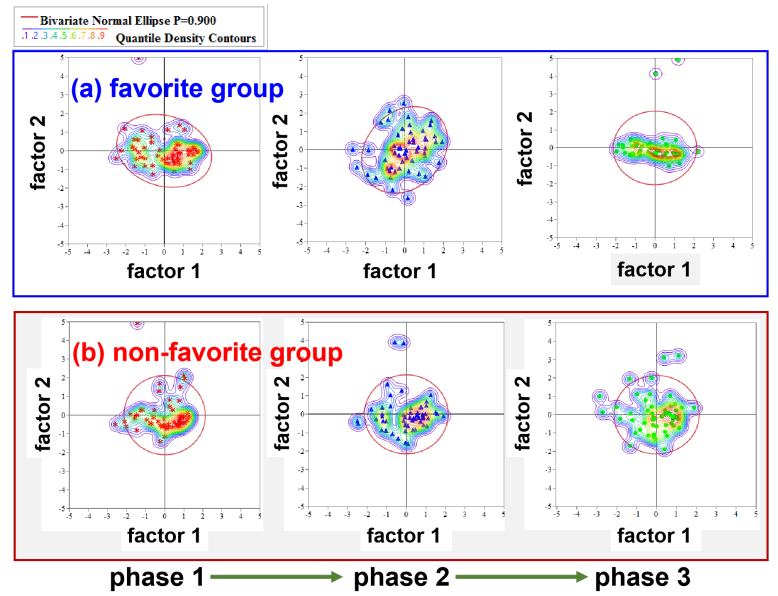

Fig. 7. Nonparametric density contours for overlapping three phases in two groups: red line shows bivariate normal ellipse: the density range was determined to be 0.9 .

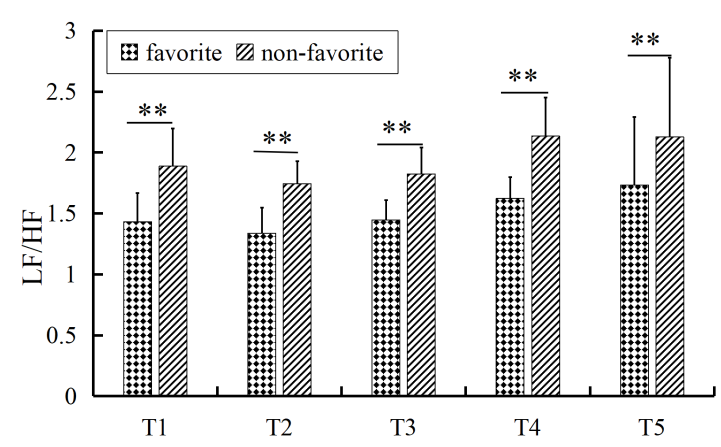

Fig. 8. Comparison of the $\mathrm{LF} / \mathrm{HF}$ ratio between favorite and non-favorite groups on every task.

expected to decrease and increase in stressful situations [8]. Therefore, fluctuations in these components are physiological evidence. Regarding the SRM-related condition, AS would be inferred as an accumulation of physiological responses, because the LF/HF ratio, a relative indicator of sympathetic nervous activity, steadily increases or shows a saturating trend during exercise [2], [20]. Therefore, the occurrence of SRM indicates would be the sign that the participant is more high favorability to stimulating music.

This approach would be suitable for localizing the temporal distribution of the SRM at each section. However, we do not yet conclude what types of ANA-related conditions could provoke this response during exercise with music. Different types of ANA-related indices would help to confirm the trigger points of SRM. Furthermore, faster music provoked responses with greater physiological fatigue than those with slower music or without music [21]. Therefore, according to the temporal distribution of SRMs (Fig. 4), we believe that the multiple time scales strategy could be suitable to evaluate and highlight the effect of distraction on physiological fatigue. Moreover, the LF/HF ratio in favorite group significantly $(* * p<0.01$ ) decreased than that in non-favorite group at every task (Fig. 8); 54 samples per group, this result was similar to that in previous reports [2], [20], [22]. As a result, the expected relaxation 
effects in the favorite group were more than those in the nonfavorite group. This suggests that greater distraction effects were due to favored music [2], [5], [6], [15], [20].

\section{B. Evaluation of distraction effects with multivariate analysis}

Borg's RPE provides a useful way to reflect how hard a cycling task feels [10]. In fact, the increase in RPE was reported to be related to both the cardiovascular status monitored by HR and local muscle fatigue during stepping exercise [8], [23], [24]. Thus, we discuss the differences of RPE between two groups in terms of biosignal interpretation with multiple regression analysis. Referring to conventional procedures, participants became physically fatigued as the cycling trial progressed, although they had different responses with multiple time scales to the stimulus music in terms of favorability. On the favorite group the TVB of estimated RPE showed a saturating trend (Fig. 5) for last tasks. It seemed to have a greater beneficial effect in relation to the distraction effect during the cycling exercise. Moreover, the $\gamma \mathrm{HR}$-eRPE in the favorite group was smaller than that in the non-favorite group and appeared an inverse correlation or no correlation phenomenon. It suggested that RPE did not increase with favorite music even though physical fatigue would be induced.

To explain this evidence, we further performed factor analysis of the ANA-related indices in three phases. From the results (Table 2, 3), we speculated that the factor 1 was dominant in both RRI and HR, and the factor 2 was dominant among spectrum indices. The simple interpretation of these factors might be concluded that the factor 1 represented the mean of HR levels and factor 2 represented the variability of HR. However, from the strong correlation between RPE and HR, factor 1 was presumed to be awareness [1], [10], [19], [25]. Recently, Schmitt et al. accurately showed how individual patterns of spectral analysis of HRV changed, diverting fatigue states without fatigue conditions. They reported that the data analysis described the clustering of different types of fatigue through mathematical proximity of HR and the dominant variables of spectral analysis [19]. Consequently, we concluded that the second factors would infer physical fatigue. From this speculation, the scores were rather concentrated on the awareness factor at phase 3 and less on the physical fatigue factor than that for other phases in the favorite group. In the non-favorite group, from the nonparametric density, the scores were probably normally distributed on the awareness and physical fatigue factors for the three phases (Fig.7). These common factors have the potential to infer the relationships between variables with a more efficient way to evaluate the distraction effects.

These results suggested that the music stimulus on the distraction effects appeared at two significant latent components that could be more directly assessed by a combination of self-reports and physiological fatigue related measures and favorability factors. Therefore, non-uniformly modeling TVB of ANA-related indices is effective for evaluating the distraction effects without taking any unnecessary evaluation time.

\section{CONCLUSION}

The expected distraction effects of music stimuli were assessed during low intensity cycling exercise, evaluating the TVB of ANA-related indices in the time and frequency domains with multiple time scales. We tried to apply nonuniformly sampling to the TVB, defining a stimulus response module observed in the time series of physiological events. Moreover, factor analysis and multiple regression analysis were adopted for properly selecting ANA-related indices to infer the psychological event, RPE. The present results showed that the multiple time scales strategy in revealing the progress of physical and mental fatigue could be of help to study the suitable approach, although further validation is required.

These findings might lead to propose a better understanding biological function with the multiple time scales. Then, other biosignals such as muscle and brain activities should be investigated more, because the physiological indices are still not enough to explain the observed distraction effects as psychological responses.

\section{ACKNOWLEDGMENT}

The authors would like to thank the all participants for participating in this experiment. This work was partially supported by National Natural Science Foundation of China (61762070, 61862048) for exploratory research.

\section{REFERENCES}

[1] V. M. Nethery, Competition between internal and external sources of information during exercise: influence on RPE and the impact of the exercise load, Journal of Sports Medicine and Physical Fitness. 42 (2), pp. $172-178,2002$

[2] K. Urakawa and K. Yokoyama, Music can enhance exercise-induced sympathetic dominancy assessed by heart rate variability, Tohoku J. Exp. Med. 3.31 (2), pp. 118-25, 2006

[3] E. Delattre, M. Garcin, L. M. Hamard and V. Billat, Objective and subjective analysis of the training content in young cyclists, Appl Physiol Nutr Metab. 42 (2), pp. 172-178, 2002.

[4] E. B. Fontes, B. P. Smirmaul, F. Y. Nakamura, G. Pereira, A. H. Okano, L. R. Altimari, J. L. Dantas, and A. C. de Moraes, The relationship between rating of perceived exertion and muscle activity during exhaustive constant-load cycling, Int J Sports Med. 31 (10), pp. 683-688, 2010.

[5] T. H. Fritz, S. Hardikar, M. Demoucron, M. Niessen, M. Demey, O. Giot , Y. Li, J. D. Haynes, A. Villringer and M. Leman , Competition between internal and external sources of information during exercise: influence on RPE and the impact of the exercise load, Proc. National Academy of Sciences USA. 5.110 (44), pp. 17784-17789, 2013.

[6] J. P. Lopes-Silva, A. E. Lima-Silva, R. Bertuzzi, M. D. Silva-Cavalcante, Influence of music on performance and psychophysiological responses during moderate-intensity exercise preceded by fatigue, Physiology $\mathrm{Be}-$ havior. 139 (2015), pp. 274-280, 2015.

[7] J. Hayano, J. A. Taylor, S. Mukai, A. Okada, Y. Watanabe, K. Takata, T. Fujinami, Assessment of frequency shifts in $R-R$ interval variability and respiration with complex demodulation, J. Appl. Physiol. 77 (6), pp. 2879-2888, 1994.

[8] T. Kiryu, I. Sasaki, K. Shibai, K. Tanaka, Providing appropriate exercise levels for the elderly, IEEE Eng Med Biol Mag. 20 (6), pp. 116-124, 2001.

[9] H. Arazi, A. Asadi, M. Purabed, Physiological and Psychophysical Responses to Listening to Music during Warm-Up and Circuit-Type Resistance Exercise in Strength Trained Men, J Sports Medicine (Hindawi Publ Corp). 2015 (389831), 2015.

[10] G. A. Borg, Psychophysical bases of perceived exertion, Medicine and Science in Sports and Exercise. 14 (5), pp. 377-381, 1982.

[11] T. Sangenya, T. X. Fujizawa , N. Nakata, Creation of sound-image scale-Quantification of the image of chord progressions with impression evaluation used, IEEE International Conference on Systems, Man and Cybernetics. pp.1905-1909, 2008. 
[12] H. Wang, S. Huang, SDNN/RMSSD as a Surrogate for LF/HF: A Revised Investigation, Modeling and Simulation in Engineering. 2012 (931943), pp. 931-943, 2012.

[13] J. Ng J, S. Sundaram, A. H. Kadish, J. J. Gold-berger, Autonomic effects on the spectral analysis of heart rate variability after exercise, The American Physiological Society. 42 (2), 297, pp. H1421-H1428, 2009.

[14] S. Akselrod, D. Gordon, F. A. Ubel, D. C. Shannon, A. C. Berger, R. J. Cohen, Power spectrum analysis of heart rate fluctuation: a quantitative probe of beat-to-beat cardiovascular control, Science. 213 (4504), pp. 220-222, 1981

[15] C. Bao, T. Kiryu, A Study on the Physiological Effects of Auditory Stimulus with Heart Rate Variability during Cycling, Proc. IEEE 4rd Global Conference on Consumer Electronics (GCCE). pp. 254 -255, 2015.

[16] T. Kiryu, A. Iijima, T. Bando, Relationships between sensory stimuli and autonomic nervous regulation during real and virtual exercises, Journal of NeuroEngineering and Rehabilitation. 4 (38), pp. 1-7, 2007.

[17] S. Bruck, P. A. Watters, The factor structure of cybersickness, Displays. 32 (2011), pp. 153-158, 2011.

[18] M. Orini, R. Bailon, R. Enk, S. Koelsch, L. Mainardi, P. Laguna, A method for continuously assessing the autonomic response to musicinduced emotions through HRV analysis, Med Biol Eng. Comput. 48 (5), pp. 423-433, 2010.

[19] L. Schmitt, J. Regnard, G. P. Millet, Monitorin Fatigue Status with HRV Measures in Elite Athletes: An Avenue Beyond RMSSD?, Frontiers Physiology. 6 (343), pp. 1-3, 2015.

[20] S. Yamashita, K. Iwai, T. Akimoto, Effects of music during exercise on RPE heart rate and the autonomic nervous system, Journal of Sports Medicine and Physical Fitness. 46 (3), pp. 425-430, 2006.

[21] J. Wanterhouse, P. Hudson, B. Edwards, Competition between internal and external sources of information during exercise: influence on RPE and the impact of the exercise load, Scand. J. Med. Sci. Sports. 20 (4), pp. 662-669, 2010 .

[22] M. Iwanaga, A. Kobayashi, C. Kawasaki, Heart rate variability with repetitive exposure to music, Biological Psychology. 70 (2005), pp. 61-66, 2005.

[23] D. D. Egorova, Y. E. Kazakov, and V. S. Kublanov, Principal Components Method for Heart Rate Variability Analysis, Biomedical Engineering. 48 (1), pp. 37-41, 2014.

[24] S. W. Chen , J. W. Liaw, Y. J. Chang, L. L. Chuang, C. T. Chien , Combined heart rate variability and dynamic measures for quantitatively characterizing the cardiac stress status during cycling exercise, Comput Biol Med. 63 (2015), pp. 133-142, 2015.

[25] S. C. Segerstrom, L. S. Nes, Heart Rate Variability Reflects SelfRegulatory Strength, Effort, and Fatigue, Association for Psychological Science. 18 (3), pp. 275-281, 2007.

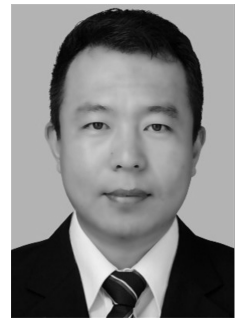

Caijilahu Bao Caijilahu Bao received the B.S. degree in Information and Computing Science from Inner Mongolia University for Nationalities , China, in 2007. The M.E. and Dr. Eng. degree in Human Sciences and Assistive Technology of Electrical and Information Engineering from Niigata University, Niigata, Japan, in 2013 and 2020. He joined the Inner Mongolia University of Technology, in 2021, where he is currently a Lecturer with the College of Data Science and Application. His research interests include the autonomic nervous biomedical engineering, the biosignal processing, speech recognition and natural language processing.

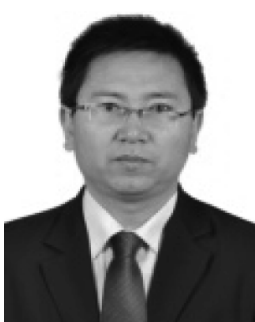

Zhiqiang Ma Zhiqiang Ma received the B.E. degree in computer application technology from Hohai University, Nanjing, China, in 1995, and the M.E. degree in computer science and technology from Beijing Information Science and Technology University, Beijing, China, in 2007. He joined the Inner Mongolia University of Technology, in 1995, where he is currently a Professor with the College of Data Science and Application. He is also a Reviewer of the Journal of Chinese Information Processing, IEEE Access, and the International Computer Frontier Conference. His research interests include speech recognition and natural language processing, with a special focus on emotional text generation.

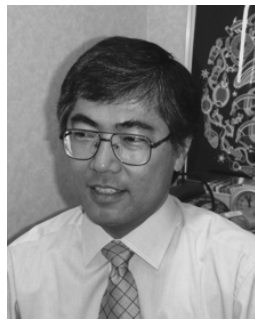

Tohru Kiryu Tohru Kiryu (M'81) received the B.E. and M.E. degrees in electronics engineering from Niigata University, Niigata, Japan, in 1975 and 1977, respectively, and the Dr. Eng. degree in computer science from the Tokyo Institute of Technology, Tokyo, Japan, in 1985.,From 1977 to 1978, he was an Assistant at the School of Dentistry of Niigata University. From 1978 to 1995 , he was with the Department of Information Engineering, Niigata University. Since 1995, he has been with the Graduate School of Science and Technology, Niigata University, as a Professor. From June 1990 to March 1991, he studied at the NeuroMuscular Research Center at Boston University as a Visiting Scientist. He has been working on biosignal processing, especially for understanding physical activity from myoelectric signals and heart rate variability during exercise and rest in field experiments. His current research interests include fatigue evaluation at a required time and place by a wearable measurement and Internet technology.,Dr. Kiryu is a Member of the International Society of Electrophysiology and Kinesiology; the Institute of Electronics, Information, and Communication Engineers of Japan; the Japanese Society of Medical and Biological Engineering; and the Japan Society of Biomechanisms. 